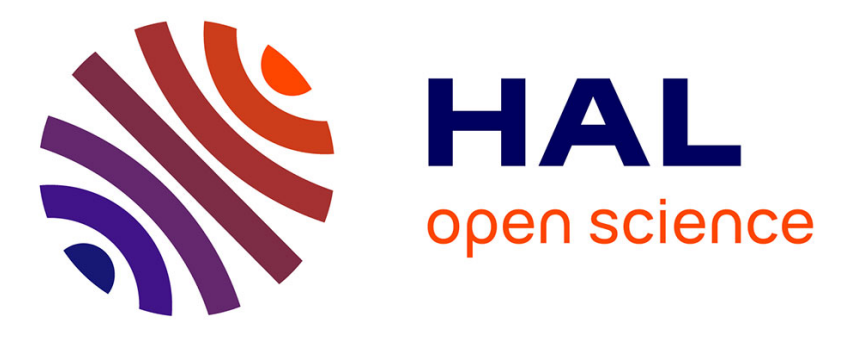

\title{
Pain perception and detailed visual pain mapping in breast cancer survivors
}

\author{
Sebastian M. Jud, Peter A. Fasching, Christian Maihöfner, Katharina \\ Heusinger, Christian R. Loehberg, Reinhard Hatko, Claudia Rauh, Hiba Bani, \\ Michael P. Lux, Matthias W. Beckmann, et al.
}

\section{To cite this version:}

Sebastian M. Jud, Peter A. Fasching, Christian Maihöfner, Katharina Heusinger, Christian R. Loehberg, et al.. Pain perception and detailed visual pain mapping in breast cancer survivors. Breast Cancer Research and Treatment, 2009, 119 (1), pp.105-110. 10.1007/s10549-009-0485-z . hal-00535388

\section{HAL Id: hal-00535388 https://hal.science/hal-00535388}

Submitted on 11 Nov 2010

HAL is a multi-disciplinary open access archive for the deposit and dissemination of scientific research documents, whether they are published or not. The documents may come from teaching and research institutions in France or abroad, or from public or private research centers.
L'archive ouverte pluridisciplinaire HAL, est destinée au dépôt et à la diffusion de documents scientifiques de niveau recherche, publiés ou non, émanant des établissements d'enseignement et de recherche français ou étrangers, des laboratoires publics ou privés. 


\title{
Pain perception and detailed visual pain mapping in breast cancer survivors
}

\author{
Sebastian M. Jud · Peter A. Fasching · Christian Maihöfner · Katharina Heusinger • \\ Christian R. Loehberg - Reinhard Hatko - Claudia Rauh - Hiba Bani · \\ Michael P. Lux · Matthias W. Beckmann · Mayada R. Bani
}

Received: 22 January 2009/Accepted: 16 July 2009/Published online: 30 July 2009

(C) Springer Science+Business Media, LLC. 2009

\begin{abstract}
Chronic pain and neural irritation after breast surgery and radiation are still relevant sequelae of the treatment. Pain quantification and localization in patient groups are difficult to standardize. In order to quantify and localize pain in a group of breast cancer patients, a Javabased program was developed to visualize the frequency of pain in "pain maps." A questionnaire with structured questions on the perception of pain included pictograms of a body to mark possible pain areas. A group of 343 breast cancer survivors completed the questionnaires. The image information was digitalized and processed using a Java applet. Gray-scale summation pictures with numbers from " 0 ," indicating black ( $100 \%$ pain), to " 255 ," indicating white ( $0 \%$ pain), were generated. The visualization of pain by creating pain maps revealed the location of pain in breast cancer survivors on pictograms of the body. Analyzing the total number of pixels, in which pain was stated, made it possible to compare pain areas in several subgroups, showing that patients after mastectomy versus breast-conserving therapy (3,011 vs. 2,224 pixels), and patients with lymphedema versus patients without lymphedema $(3,010$
\end{abstract}

S. M. Jud · P. A. Fasching $(\bowtie) \cdot$ K. Heusinger .

C. R. Loehberg - C. Rauh · H. Bani - M. P. Lux ·

M. W. Beckmann · M. R. Bani

Department of Gynecology and Obstetrics, University Breast

Center for Franconia, Erlangen University Hospital,

Universitaetsstrasse 21-23, 91054 Erlangen, Germany

e-mail: peter.fasching@uk-erlangen.de

\section{Maihöfner}

Department of Neurology, Erlangen University Hospital,

Erlangen, Germany

R. Hatko

Department of Artificial Intelligence and Applied Computer Science, University of Würzburg, Würzburg, Germany vs. 2,239 pixels), have larger pain areas. This study presents a method of visualizing pain areas and assigning them to a pictogram of the body in a sample of breast cancer patients. The method is easy to use and could help generate pain maps in several types of disease.

Keywords Breast cancer · Digital imaging · Postmastectomy pain · Pain mapping

\section{Introduction}

The management of breast cancer involves surgery, radiotherapy, chemotherapy, and antihormonal medication. All of these therapies have specific side effects and sequelae. This article concerns with a methodology to document, visualize, and compare pain in groups of breast cancer patients. Chronic pain after surgical treatment and radiation, mostly referred to as postmastectomy pain (PMP), is common and the prevalence is $\sim 20-43 \%$ [1-4].

Most studies used questionnaires either to describe the frequency or the quality of the pain: The characteristics of this pain are mainly described diversely as consisting of lancinating pain, paresthesia, dysesthesia, weird sensations, hyperalgesia or allodynia, edema, muscle weakness, and skin irritations [5], indicating that the symptoms are related to nerve injury or dysfunction. Additionally sensitization processes may occur in the peripheral and central nociceptive system, leading to primary and secondary hyperalgesia. In addition, the occurrence of painful hypoalgesic areas indicates deafferentation pain in some patients. Pain after breast cancer treatment can therefore be described as a neuropathic, chronic pain condition [2, 6, 7]. Treatment effects as well have been measured mainly with questionnaires but their harmonization and comparison seems to be 
difficult [8], as timing, pain intensity, pain quality, and neuropathic components have to be taken into consideration.

Accurate and objective assessment of pain in patients with pain after breast cancer therapy is therefore of paramount importance in understanding the underlying pathophysiology and guiding appropriate treatment. As in other chronic pain states, one important step can be the mapping of painful areas [9]. Unfortunately, a systematic tool has not previously been available for mapping pain symptoms visually. To provide the clinician with more detailed support during the follow-up in breast cancer survivors for detecting and identifying pain in breast, cancer patients the present study aimed to establish a computer-based routine for visualizing pain, identifying high-frequency and low-frequency locations. In addition, ways of comparing the extent and level of pain among subgroups of breast cancer patients were explored.

\section{Methods}

Patients and questionnaire

A cross-sectional survey with three aims was conducted in the outpatient follow-up department of our breast center between April 2004 and November 2005. All of the patients provided written informed consent, and the study was approved by the ethics committee at the University of Erlangen. Two of the three aims (lymphedema prevalence and cosmetic satisfaction with the breast surgery) have been reported elsewhere $[10,11]$. This article is concerned with the visual assessment of pain perception, which the patients documented on a questionnaire including pictograms (Fig. 1) in which the patients were asked to mark the area of pain, regardless of pain intensity. Common patient characteristics were obtained from the patients' records, making it possible to construct pain maps for subgroups of breast cancer patients.

\section{Digitalization of pain mapping information}

Using an Intuos3 professional pen tablet (Wacom Europe Ltd, Krefeld, Germany) and the ImageJ picture editing program (Research Services Branch, National Institute of Mental Health, Bethesda, MD, USA), areas of pain and no pain were digitized into a tabulator-separated black/white text-file with a size of $876 \times 620$ pixels, with values 0 (representing black indicating pain areas) or 255 (representing white, indicating no pain areas). The result was a 32-bit text-file image. To ensure consistency in the imagedigitizing procedure, the position of the pictograms on the pen tablet was standardized, they were saved with a unique number, and the number of the picture was added. Pictures in which areas were not marked were not digitized.

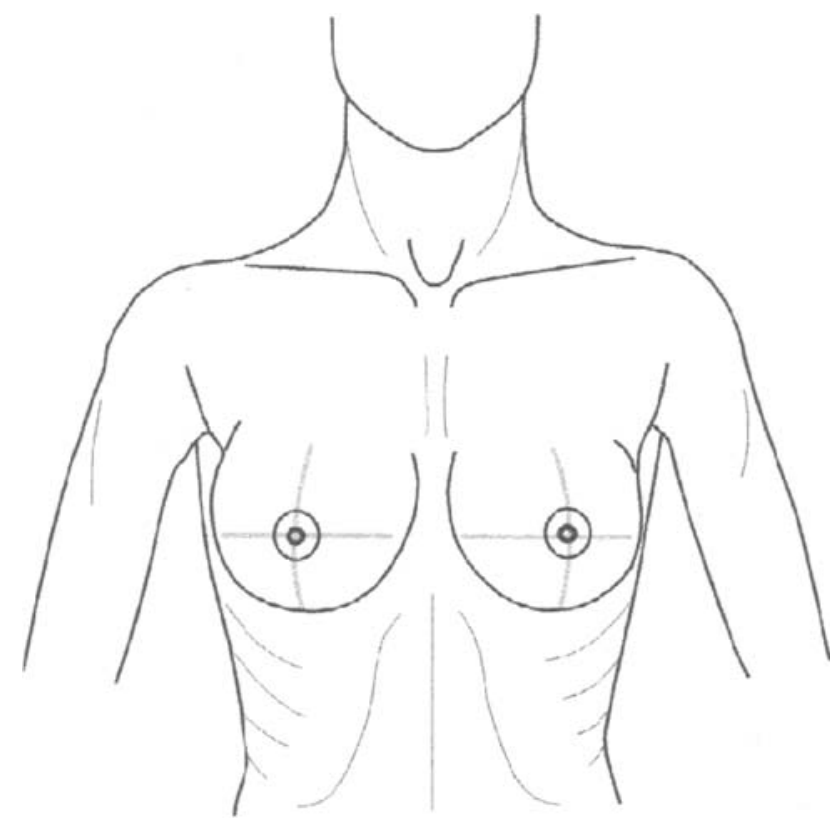

Fig. 1 Front-view pictogram

Computing process and picture calculations

Summation pictures were created as an overlay of each individual pixel with a gray scale representing the frequency of patients stating pain located at the specific pixel. The summation pictures were created using a Java-based program (Java, Sun Microsystems, Inc., Santa Clara, CA, USA; programmed by R.H.), which displays patient data and opens the patient-specific tabulator-separated matrix. Each cell in the matrix is processed into a new tabulatorseparated file named after the corresponding picture by adding it to the previous value of the cell and calculating the mean value. The subsequent picture is processed in the same way. This procedure produces a processed gray-scale image with values from 0 to 255 for each query. A value of 0 represents the color black, indicating that $100 \%$ of all patients stated pain at this specific location and a value of 255 represents the color white, indicating that $0 \%$ of all patients stated pain at this specific location. Summarized, the blacker an area is, the larger the number of cumulative pain events occurring in it. If a patient did not mark an area and stated that she had no pain, the applet automatically added the value 255 (white), indicating "no pain/irritation."

To obtain a better impression of the pictures and to standardize them, the following values were defined: the pain value $(\mathrm{PV})$ is the value of one pixel, with a scale from 0 , representing black $(=100 \%$ of patients had pain/sensory irritation at this specific location), to 255 , representing white $(=0 \%$ of patients had pain/sensory irritation at this specific location). The pain area (PA) is the area, measured as the number of pixels, of the total picture with any $\mathrm{PV}<250$. 
mean_PV $=\sum_{1}^{876 \times 620}($ pixel_value $)$

$\mathrm{PA}=\sum($ pixel $\mid$ pixel_value $<250)$

Comparisons of the PA were carried out using student's $t$-test, with the SPSS software package, version 15.0.

\section{Results}

A total of 343 patients marked the pain area in the pictogram. The characteristics of these patients are listed in Table 1.

The gray scales in the computed pictures represent the percentage of patients who stated that they had pain at each specific location on the pictogram. The pictures represent an overlay of all the pain areas that were marked by all of the patients.

Figure 2 shows separate pain maps for patients who had breast cancer either on the left side or the right side. Generally, four different locations can be visually differentiated. The first area is the breast itself, which appears to be affected in a substantial proportion of patients. The second area is the axilla. This area is rather dark, indicating that most of the patients experienced pain in this area. The third area is the upper chest wall, and a fourth area can be seen over the sternum.
Several subgroups were compared in an exploratory analysis, but without differentiating between cancers of the left and right breast, resulting in images that show the pain locations marked on both sides (Figs. 3, 4, 5).

Influence of surgery on pain

When the images from patients who underwent breastconserving surgery (BCS) are compared with those from patients with mastectomy, it appears that patients with mastectomy have an additional field of pain over the chest wall. For interpretation, it should be mentioned that patients who underwent mastectomy received a pictogram that did not show the breast on the affected side, but rather a scar on the chest wall (pictogram not illustrated here). The mean marked pixels (PA) differed significantly, with patients after BCS marking 2,224.6 $( \pm 2,659.3)$ pixels and patients after mastectomy marking 3,010.7 $( \pm 2,905.3)$ pixels $(P=0.018)$.

\section{Influence of lymphedema on pain}

The pictures from patients with and without lymphedema (Fig. 3) differ with regard to the size of the pain area and the percentage indicating pain in specific areas. Both of these qualities were poorer in patients with lymphedema. Particularly in the axilla, the pictures indicate a higher percentage
Table 1 Demographic data for the patients
$B C T$ breast-conserving therapy, $M R M$ modified radical mastectomy, $N A$ not applicable, $P A$ pain area

${ }^{\text {a }}$ Only tested in women with BCT

b Twelve patients were operated with sentinel node biopsy, the rest with conventional axillary dissection

\begin{tabular}{lclc}
\hline Characteristic & $N$ & PA & $P$ value \\
\hline Total no. of questionnaires analyzed & 343 & NA & NA \\
Age (years) & $52.1( \pm 12.4)$ & NA & NA \\
Surgical procedure & & & 0.018 \\
$\quad$ BCT & 248 & $2,224.6( \pm 2,659.3)$ & \\
MRM & 95 & $3,010.7( \pm 2,905.3)$ & \\
Total & 343 & $2,442.3( \pm 2,748.0)$ & \\
Tumor size & & & \\
T1 & 148 & $2,264.4( \pm 2,308.8)$ & \\
T2-4 & 80 & $2,223.6( \pm 3,409.6)$ & \\
Total & 228 & $2,250.1( \pm 2,738.3)$ & \\
Lymphedema & & & \\
No & 170 & $2,239.0( \pm 2,089.6)$ & \\
Yes & 118 & $3,010.3( \pm 3,760.4)$ & \\
Total & 288 & $2,555.0( \pm 2,912.1)$ & \\
Nodal status ${ }^{\mathrm{b}}$ & & & \\
Negative & 201 & $2,476.7( \pm 2,612.3)$ & 0.881 \\
Positive & 105 & $2,528.5( \pm 3,299.9)$ & \\
Total & 306 & $2,494.5( \pm 2,861.6)$ & \\
Adjuvant radiotherapy & & $2,195.7( \pm 2,370.8)$ & \\
No & 46 & $2,482.7( \pm 2,819.1)$ & \\
Yes & 293 & & \\
Total & 339 & & \\
\hline
\end{tabular}


(a)

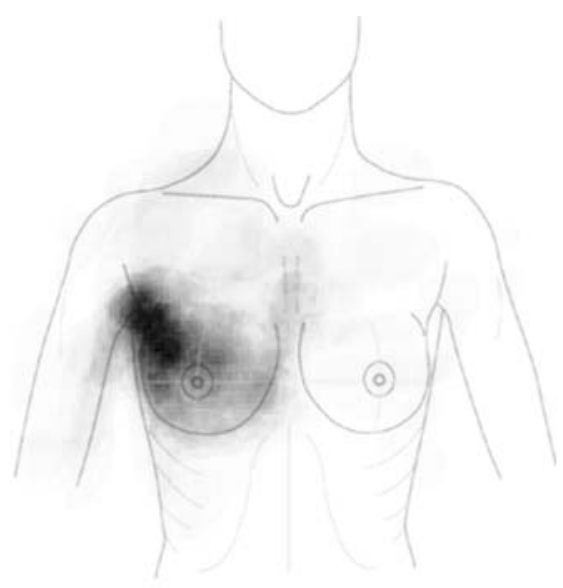

$100 \%$

$50 \%$

$0 \%$

(b)

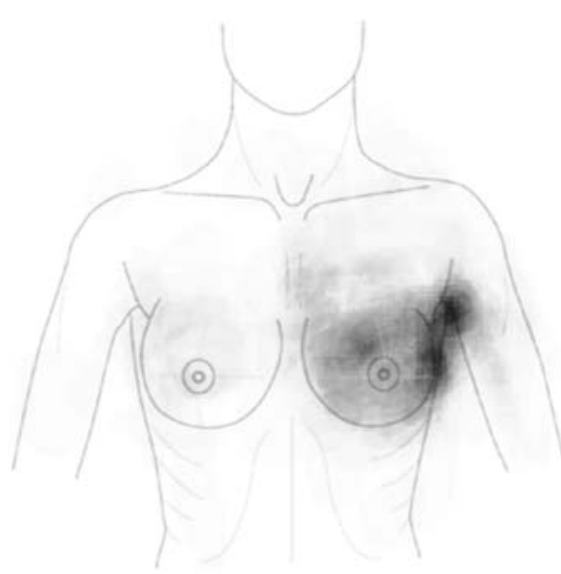

$100 \%$

$50 \%$

$0 \%$

Fig. 2 a Summation picture for patients with breast cancer on the left side (the bar below indicates the percentage of patients who stated that they had pain in the specific location). b Summation picture for patients with breast cancer on the right side (the bar below indicates the percentage of patients who stated that they had pain in the specific location)

of patients experiencing pain in this area. Comparison of the pain area between the two groups showed significant differences $(P=0.027)$. Patients with lymphedema marked an area (PA) of $3,010.3( \pm 3,760.4)$ pixels, versus $2,239.0$ $( \pm 2,089.6)$ pixels in women without lymphedema.

Tumor size, nodal status, and adjuvant radiotherapy did not have any influence on the extent of the area of pain marked. Other variables checked (e.g., chemotherapy or antihormonal treatment) did not have an effect either (data not shown). (a)

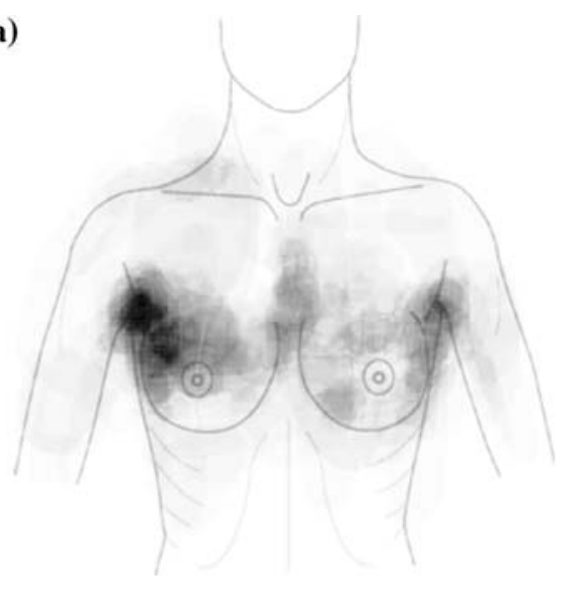

$100 \%$

$50 \%$

$0 \%$

(b)

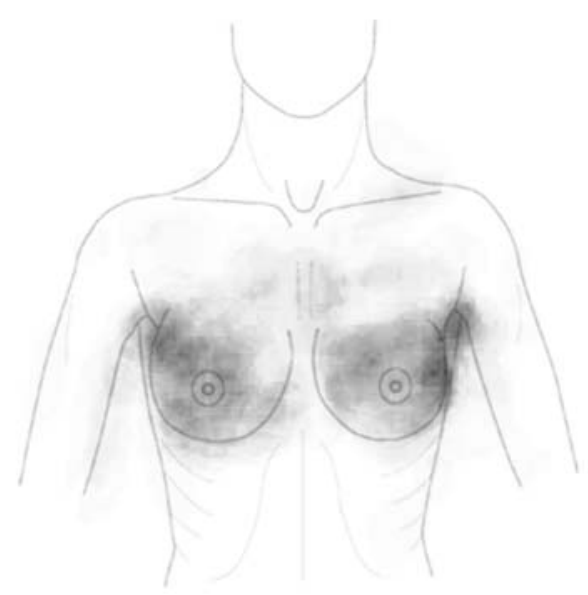

$50 \%$

$0 \%$

Fig. 3 a Summation picture for patients with lymphedema (the bar below indicates the percentage of patients who stated that they had pain in the specific location). b Summation picture for patients without lymphedema (the bar below indicates the percentage of patients who stated that they had pain in the specific location)

\section{Discussion}

This is the first description of localized pain mapping in a large cohort of breast cancer survivors. The aim was to visualize the pain area and assess the percentage of patients who experience pain. The visualized pain area and the displayed percentage of patients were in agreement with experience in clinical practice. Using the method described in this paper, pain as an outcome measure in breast cancer survivors can now be compared not only with the patients' 
(a)

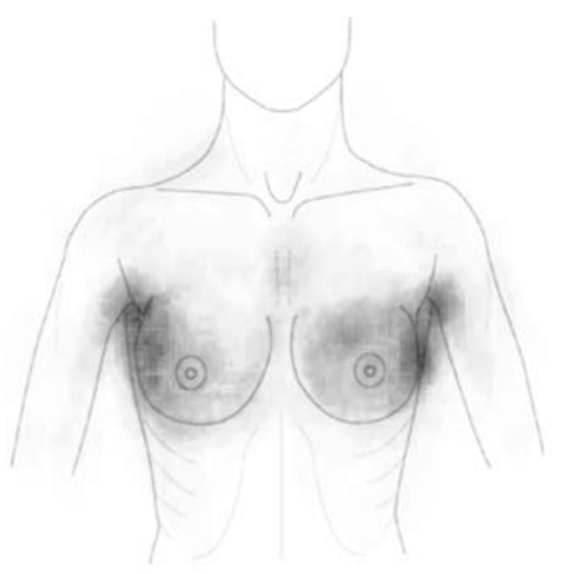

$100 \%$ $50 \%$

$0 \%$

(b)

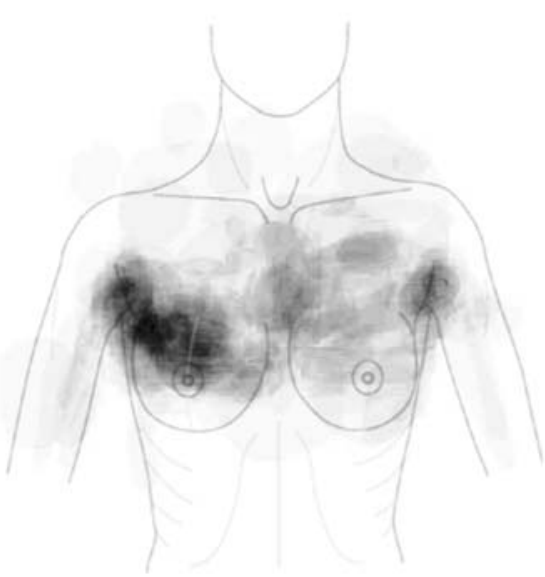

$100 \%$

$50 \%$

$0 \%$

Fig. 4 a Summation picture for patients who underwent breastconserving surgery (the bar below indicates the percentage of patients who stated that they had pain in the specific location). b Summation picture for patients who underwent mastectomy. Mastectomy was more often done on the right side in our cohort (the bar below indicates the percentage of patients who stated that they had pain in the specific location)

reported quantitative pain assessment on visual analogue scales, for example, but also with a visual assessment.

So far as we are aware, only one other study has been published on pain mapping [9]. The study included women who were referred to a facial pain clinic. The authors visualized the pain by analyzing a pictogram of the whole body. The patients had to mark the area of pain, as in the present study. This pictogram was divided into approximately 1,900 "cells." After this, visualization was (a)

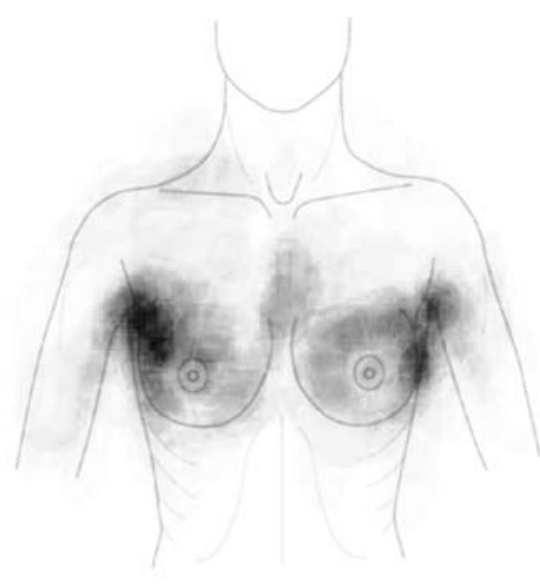

$100 \%$

$50 \%$

$0 \%$

(b)

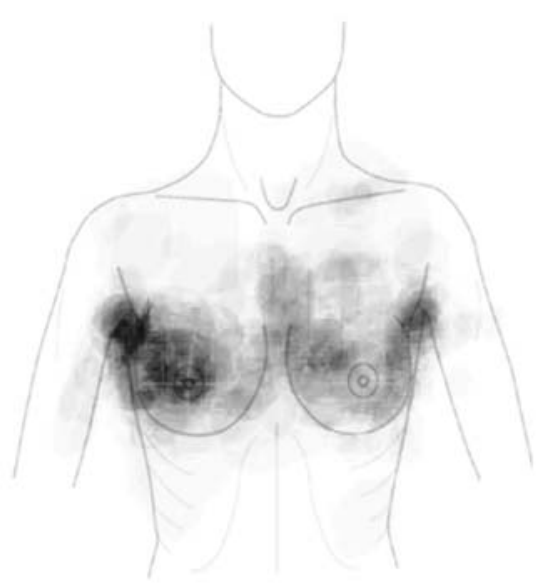

$100 \%$

$50 \%$

$0 \%$

Fig. 5 a Summation picture for patients with pT1 tumors (the bar below indicates the percentage of patients who stated that they had pain in the specific location). b Summation picture for patients with pT2 or pT3 tumors (the bar below indicates the percentage of patients who stated that they had pain in the specific location)

achieved by projecting the number of patients over the pictogram in a three-dimensional picture, providing an idea of where the patients have pain and where they do not. However, due to the three-dimensional presentation method and oblique presentation of the pain visualization, exact localization of the pain was not possible. This issue was addressed in the present study and solved by presenting the percentage of patients using gray scales, making it possible to present a planar image.

Although the comparison of subgroups was exploratory here, there are some findings worth discussing. A higher 
incidence of chronic pain was observed in the modified radical mastectomy (MRM) patients in comparison with the BCS group, as was contrary reported in the study by Tasmuth et al. [5]. The findings for the radiotherapy versus no radiotherapy groups differed as well in this study in comparison with others. While there was no difference in the present study between the pain areas in the radiotherapy group and those in the group without radiotherapy, Tasmuth et al. [5] reported a difference, with radiotherapy patients experiencing more pain. When $\mathrm{T} 1$ and $\mathrm{T} 2-4$ tumors were compared, it was found that tumor size had no effect on the incidence of pain. The lymphedema group had a larger pain area than the nonlymphedema group. This confirms the results described in other studies [12].

The incidence of pain symptoms can be illustrated using the method described in this paper. As weakness of the presented method, however, intensity measures are not taken into account in this initial report. In principle, it would be possible to include data on the intensity of the pain in the model, possibly based on a color-coding system. However, this would mean that patients would have to indicate the pain intensity in every marked area, which was not requested in this study. Furthermore, the presented illustrations only refer not to further specified pain and not to other special qualities of pain or sensory irritation. Different pain qualities would have to be documented on different pictograms, which then could be matched to each other. But this was not part of the study reported here.

There are several ways in which this method could be helpful in understanding and comparing the nature of pain in breast cancer. The advantage of using pain maps is that the pain can be easily localized and the area on a body surface in a patient group can be measured and be compared within patient groups. Especially, pain that occurs in special patterns on the body surface could be attributed to organs, dermatomes, or joints. For example, postmenopausal hormone receptor-positive breast cancer patients are commonly treated with an aromatase inhibitor. One of the main side effects compromising the quality of life in these patients is musculoskeletal pain sensations, the pathogenesis of which is still unclear. Comparing a pain map for these patients with one for patients with other diseases might reveal similarities in a measurable and comparable way. Other diseases that could benefit from this type of visualization might include endometriosis, migraine, fibromyalgia, and other chronic pain syndromes.

Acknowledgments The authors are grateful to Gassan Bani, MD, who helped substantially in the data management process for this study. Peter A. Fasching is funded by the Dr. Mildred Scheel Stiftung, Deutsche Krebshilfe e.V.

\section{References}

1. Carpenter JS, Andrykowski MA, Sloan P et al (1998) Postmastectomy/postlumpectomy pain in breast cancer survivors. J Clin Epidemiol 51:1285-1292

2. Macdonald L, Bruce J, Scott NW et al (2005) Long-term followup of breast cancer survivors with post-mastectomy pain syndrome. Br J Cancer 92:225-230

3. Stevens PE, Dibble SL, Miaskowski C (1995) Prevalence, characteristics, and impact of postmastectomy pain syndrome: an investigation of women's experiences. Pain 61:61-68

4. Smith WC, Bourne D, Squair J et al (1999) A retrospective cohort study of post mastectomy pain syndrome. Pain 83:91-95

5. Tasmuth T, von Smitten K, Hietanen P et al (1995) Pain and other symptoms after different treatment modalities of breast cancer. Ann Oncol 6:453-459

6. Ferguson A (2007) Discovery of neuropathic pain following breast surgery. Br J Nurs 16:102

7. Kehlet H, Jensen TS, Woolf CJ (2006) Persistent postsurgical pain: risk factors and prevention. Lancet 367:1618-1625

8. Tatrow K, Montgomery GH (2006) Cognitive behavioral therapy techniques for distress and pain in breast cancer patients: a metaanalysis. J Behav Med 29:17-27

9. Turp JC, Kowalski CJ, O'Leary N et al (1998) Pain maps from facial pain patients indicate a broad pain geography. J Dent Res 77:1465-1472

10. Bani HA, Fasching PA, Lux MM et al (2007) Lymphedema in breast cancer survivors: assessment and information provision in a specialized breast unit. Patient Educ Couns 66:311-318

11. Bani MR, Beckmann K, Engel J et al (2008) Correlates of the desire for improved cosmetic results after breast-conserving therapy and mastectomy in breast cancer patients. Breast 17(6):640-645

12. Hamner JB, Fleming MD (2007) Lymphedema therapy reduces the volume of edema and pain in patients with breast cancer. Ann Surg Oncol 14:1904-1908 